\title{
Variabilidad en el florecimiento de procedencias de Eucalyptus cladocalyx en la Región de Coquimbo
}

\author{
Freddy Mora ${ }^{1}$, Sandra Perret ${ }^{2}$, Carlos Alberto Scapim¹, Elías Nunes Martins ${ }^{1}$ \\ y María Paz Molina ${ }^{2}$ \\ ${ }^{1}$ Centro de Ciencias Agrarias, Universidade Estadual de Maringá. \\ Av. Colombo, 5790, Bloco 05 (PGM), CEP 87020-900. Maringá, PR, Brasil. \\ ${ }^{2}$ Instituto de Investigación Forestal, Ministerio de Agricultura de Chile. \\ Huérfanos 554, Santiago, Chile.
}

\begin{abstract}
F. Mora, S. Perret, C.A. Scapim, E.N. Martins, and M.P. Molina. 2007. Source-dependent blooming variability of Eucalyptus cladocalyx in the Region of Coquimbo, Chile. Cien. Inv. Agr. 34(2):131-139. This work aimed to study the variability in flowering of five populations of Eucalyptus cladocalyx established in the administrative region of Coquimbo, northern Chile. Flowering was recorded as a binary response trait in 30-month-old trees. Then, at first, a generalized linear model was fitted for analyzing provenance effect. Subsequently, genetic variability was studied within the best provenance by Bayesian inference via Gibbs sampling. Initially, two sites were considered: Caracas and Tunga Norte, in the littoral and interior area of the Choapa Province, respectively. In the littoral area, only $1.3 \%$ of the trees evidenced flowering events, while in Tunga Norte, this value was $23.43 \%$. For this reason, only interior site was considered. The provenance effect was significant $(\mathrm{p}<0.01)$, with Cowell (codified FDI20411) being the population that evidenced a relative better flowering response, although it not was different of the trees from national plantations (FDI-Illapel). However, trees from a local population evidenced a high variability, having a mean response similar to other provenances. The flowering trait analyzed within the Cowell population, the selected provenance, was shown to be highly heritable $\left(\mathrm{h}^{2}=52.3 \%\right)$. Predicted genetic gain when considering two different breeding strategies varied from 36 to $40 \%$. According to these results, it is possible that farmers can recover establishment costs of $E$. cladocalyx plantations as soon as possible whenever the selected trees are used for apicultural aims.
\end{abstract}

Key words: Arid conditions, flowering events, genetic gain, improvement, multipurpose trees, provenances.

\section{Introducción}

Los eucaliptos (Eucalyptus spp.) se plantan extensivamente como árboles ornamentales, y producción agroforestal más allá de su distribución natural en Argentina, Brasil, Chile, España, Estados Unidos, Etiopía, Nueva Zelandia, Portugal y Sudáfrica (Ruthrof et al., 2003). En Chile, las especies E. globulus y E. nitens se reportan como las más importantes del punto de vista de la industria forestal (Bonilla

Recibido 20 de octubre 2006. Aceptado 06 de marzo 2007 ${ }^{1}$ Dirigir correspondencia a Freddy Mora: fmora@universiabrasil.net. et al., 2000; Bonomelli y Suárez, 1999; Geldres y Schlatter, 2004; Geldres et al., 2006).

El potencial de algunas especies de Eucalyptus especificamente para las regiones áridas y semi-áridas se debe a su tolerancia a suelos salinos y a extensos períodos con déficit hídrico (Hanks et al., 1994). En Chile, Coquimbo es una zona árida mediterránea con potencial para el establecimiento de plantaciones forestales que toleran continuos períodos de sequía (Montenegro et al., 2003; Squeo et al., 2006). Esta región posee una gran diversidad florística, superior a otras regiones de Chile, la que es utilizada por Apis mellifera. La 
producción de miel constituye una alternativa económica de comprobada rentabilidad para los agricultores de esta zona (Montenegro et al., 2003). En regiones áridas la producción de miel depende, entre otros factores, de la abundancia floral existente y de la distribución de ésta a través del año (Porter, 1978). Por ello, es posible complementar al valioso recurso nativo existente, a través de programas operacionales de plantación que consideren alternativas forestales de comprobada aptitud melífera, en sitios considerados usualmente marginales.

Montenegro et al., (2003) estudiaron la composición polínica en diferentes muestras de mieles recolectadas de apiarios de la región mediterránea árida de Chile. De acuerdo con los resultados de estos estudios se encontró presencia de polen de Eucalyptus spp. en aproximadamente un $38 \%$ del total de apiarios, correspondiendo en su totalidad a E. globulus. Esta especie no es apta para zonas con baja disponibilidad hídrica (Mora, 2004). Por lo tanto, es probable que se encuentre en los bordes de ríos, quebradas o valles. Estudios previos indican que $E$. globulus tiene un mejor rendimiento silvícola en las regiones del sur de Chile, donde la precipitación media anual varia entre 500 y 1200 mm (Prado y Barros, 1989).

Por otra parte, E. cladocalyx es una especie de comprobada aptitud para crecer en ambientes áridos (Gleadow et al., 1998; Woodrow et al., 2002; Mora, 2006). Además, es relativamente resistente a Phoracantha semipunctata, insecto introducido en Chile que afecta plantaciones de Eucalyptus distribuidas entre la III y X Regiones de Chile (Hanks et al., 1994; Jorquera, 1998). Observaciones previas de los agricultores de la región de Coquimbo constataron que los eucaliptos florecen en un período crítico del punto de vista de la oferta floral. La floración ocurre aproximadamente entre diciembre y abril, períodos caracterizados por la ausencia total de precipitaciones.

Las características principales de la miel monofloral originada de E. cladocalyx, se han reportadopreviamente(ManikisyThrasivoulou, 2001) y se encuentra así indicado en el directorio de los principales recursos melíferos monoflorales del mundo, de la Asociación
Internacional de Investigación Apícola (Crane et al., 1984). Entre otras características se destaca el adecuado índice de granulación y la escasa tendencia a la granulación que presentan estas mieles.

Adicionalmente, el sector rural regional de Coquimbo necesita cubrir la demanda maderera y bioenergética (Mora y Perret, 2002). Por lo tanto, el establecimiento de plantaciones de eucaliptos con estos fines, en sitios marginales, permitiría disminuir la presión por explotar los recursos nativos existentes. Así se evitaría la explotación de este valioso recurso para la apicultura regional (Avila et al., 1993; Fuentes et al., 1995; Montenegro et al., 2003; Mora, 2004).

El establecimiento de ensayos genéticos que incluyen la introducción de nuevas poblaciones representa el primer paso para los programas de mejoramiento, y son útiles para identificar los mejores recursos genéticos para áreas determinadas. En general, en los ensayos de procedencias se desea encontrar una significativa variación genotípica para diversas características de interés económico, la cual puede ser investigada $e x$-situ, permitiendo la identificación y utilización de árboles superiores para propósito múltiple (Mulatya et al., 2002).

Con el propósito de potenciar sus actuales actividades económicas, las comunidades y empresas agrícolas de la Región de Coquimbo, motivados por las características favorables que posee E. cladocalyx, en conjunto con instituciones del Ministerio de Agricultura, Gobierno de Chile, establecieron ensayos genéticos con material proveniente de diversos sectores donde la especie crece en forma natural. Este trabajo tuvo como objetivo seleccionar árboles con florecimiento precoz que eventualmente permita conseguir un rápido retorno de la inversión forestal. Así, el presente estudio se basó en registros del florecimiento medidos en E. cladocalyx de 30 meses de edad, en dos ensayos establecidos en la Región de Coquimbo. Además, tuvo como objetivos analizar ex-situ la variabilidad entre procedencias de la especie y generar una base de datos agroforestal para el inicio de un programa de mejoramiento de E. cladocalyx en la región árida del norte de Chile. 


\section{Materiales y métodos}

\section{Descripción de los ensayos y sitios de estudio}

Los ensayos se plantaron en septiembre de 2001 en la Comunidad Agrícola Tunga Norte (31 $38^{\circ}$ Lat. S; 71 ${ }^{\circ} 19^{\prime}$ Long. O; $297 \mathrm{~m}$ de altitud; 243,7 $\mathrm{mm}$ de precipitación media anual; $15^{\circ} \mathrm{C}$ de temperatura media anual) y en la Hacienda Agrícola Caracas $\left(31^{\circ} 55^{\prime}\right.$ Lat. S.; $71^{\circ} 27^{\prime}$ Long. O; $167 \mathrm{~m}$ de altitud; 201,1 mm de precipitación media anual; $14,1^{\circ} \mathrm{C}$ de temperatura media anual), ambas localidades ubicadas en la Provincia de Choapa.

Los ensayos se establecieron con cinco poblaciones en ambos sitios utilizando un diseño de bloques al azar, con 30 bloques y número variable de árboles por parcela, con árboles densamente plantados $(2 \times 3 \mathrm{~m})$. Las procedencias están siendo investigadas, entre otros aspectos, para su utilización como recursos melíferos, y en una primera instancia se buscó la selección de poblaciones que presenten un florecimiento precoz, durante la estación seca.

\section{Material biológico y colección de datos}

El lugar de origen de cuatro procedencias australianas y una población proveniente de plantaciones previamente establecidas en la región árida de Chile se presenta en el Cuadro 1. Las procedencias australianas se obtuvieron a través de la población base del CSIRO (Commonwealth Scientific and Industrial Research Organisation), Australia.
Datos del florecimiento se registraron 30 meses después de la plantación. El florecimiento de los árboles se consideró como una característica binaria, codificada como: 0, en la ausencia de formación de yemas florales, y 1, en la presencia de cualquier estado después de la formación de yemas florales (yemas florales, flores o cápsulas).

Como una primera estrategia de mejoramiento se seleccionó la mejor población para la característica de interés, y posteriormente se efectuó un análisis genético considerando un modelo mixto de árboles individuales y pedigree conformado por familias provenientes de polinización abierta.

\section{Análisis estadístico y estimación de parámetros}

Debido a la presencia de supuestos que son limitantes en el análisis de varianza convencional (Dos Santos y Mora, 2007), el presente análisis se realizó utilizando la metodología de modelos lineales generalizados (MLG) (Nelder y Wendderburn, 1972). El modelo lineal generalizado puede ser visto como una unificación de los modelos de regresión lineal y no lineal que incorporan una extensa familia de distribuciones de la variable respuesta, ya sea normal o no (Myers et al., 2002). Siguiendo este procedimiento, el florecimiento tiene una distribución perteneciente a tal familia de distribuciones (Dos Santos y Mora, 2007). La función de ligación logit se utilizó para el ajuste del modelo generalizado (Myers et al., 2002). Esta función de ligación es la canónica (función

Cuadro 1. Origen de los árboles de Eucalyptus cladocalyx probados en la región árida de Chile.

Table 1. Origin of the Eucalyptus cladocalyx genotypes that were tested under the arid region of Chile.

\begin{tabular}{|c|c|c|c|c|}
\hline Código $^{1}$ & $\begin{array}{l}\text { Lugar } \\
\text { Origen }\end{array}$ & $\begin{array}{l}\text { Latitud } \\
\text { (Lat S) }\end{array}$ & $\begin{array}{l}\text { Longitud } \\
\text { (Long O) }\end{array}$ & $\begin{array}{l}\text { Precipitación } \\
\left(\mathrm{mm} \cdot a \tilde{n} o^{-1}\right)\end{array}$ \\
\hline FDI-20388 & Remarkable & $32^{\circ} 43^{\prime} 00^{\prime \prime}$ & $138^{\circ} 06^{\prime} 00^{\prime \prime}$ & 242,8 \\
\hline FDI-20411 & Cowell & $33^{\circ} 38^{\prime} 30^{\prime \prime}$ & $136^{\circ} 40^{\circ} 58^{\prime \prime}$ & 405,0 \\
\hline FDI-20389 & Wirrabara & $33^{\circ} 06^{\prime} 10^{\prime \prime}$ & $138^{\circ} 14^{\prime} 04^{\prime \prime}$ & 256,6 \\
\hline FDI-20267 & Flinders Chase & $35^{\circ} 57^{\prime} 00^{\prime \prime}$ & $136^{\circ} 42^{`} 00^{\prime \prime}$ & 637,9 \\
\hline FDI-Illapel & Illapel IV Región & $31^{\circ} 40^{\prime} 46^{\prime \prime}$ & $071^{\circ} 14^{\prime} 55^{\prime}$ & 243,7 \\
\hline
\end{tabular}

${ }^{1}$ Los códigos corresponden a un proyecto financiado por el Fondo de Desarrollo e Innovación (FDI), cuyo organismo ejecutor fue el Instituto de Investigación Forestal (INFOR).

${ }^{\prime}$ Codes are those cited in a project performed by Instituto de Investigación Forestal (INFOR), Chile that was supported by Fondo de Desarrollo e Innovación (FDI), Chile. 
natural) para el modelo con distribución binomial. Ligaciones canónicas son usualmente preferidas debido a sus propiedades teóricas; su uso también simplifica los cálculos aritméticos. A través del procedimiento GENMOD de SAS, se realizó un análisis de desvianza (definida como una medida de ajuste del modelo estadístico) (Nelder y Wendderburn, 1972). Este análisis examina las diferencias entre las poblaciones, asumiendo un modelo de efectos fijos, ignorando el parentesco entre los árboles.

Una vez verificado un efecto significativo de las procedencias en el modelo, se estableció un análisis de contrastes ortogonales para determinar las diferencias entre cada genotipo. Para esto se utilizó la opción CONTRAST, en SAS, el cual se basó en el estadístico de la razón de verosimilitud (Mora et al., 2007).

En el análisis familiar, se consideró el ajuste de un modelo lineal mixto; considerando los efectos genéticos aditivos (valores genéticos) de los árboles individuales. Para este análisis genético, se consideró la estimación de componentes de varianza, heredabilidad individual y valores genéticos de los árboles, a través de inferencia Bayesiana vía algoritmo de Gibbs, variante de los métodos de Monte Carlo, basados en Cadenas de Markov (MCMC) (Mora y Arnhold, 2006). La heredabilidad $\left(h^{2}\right)$, o grado de control genético aditivo, se estimó según la siguiente expresión:

$$
h^{2}=\frac{\sigma_{a}^{2}}{\sigma_{a}^{2}+\sigma_{e}^{2}}
$$

Donde $\sigma_{a}^{2}$ y $\sigma_{e}^{2}$ corresponden a los componentes de varianza aditiva y residual, respectivamente.

El programa MTGSAM (Multi-Trait Gibbs Sampler for Animal Model) (Van Tassell y Van Vleck, 1996), se utilizó para conformar las distribuciones a posteriori de los parámetros, basado en un modelo con variables de distribución binomial (Van Tassell et al., 1998).

Para constatar la convergencia de las cadenas generadas por el algoritmo de Gibbs, se utilizó el método de Heidelberger y Welch (1983) disponible en la biblioteca CODA (Convergence Diagnostics and Output Analysis), desarrollado por Cowles et al. (1995).

\section{Resultados y discusión}

Diferencias estadísticas significativas $(\mathrm{p}=$ 0,0001 ) se obtuvieron para el efecto del sitio. En la Hacienda Agrícola Caracas, sólo un 1,33\% de los árboles presentaron eventos de florecimiento a los 30 meses de edad; mientras que en Tunga Norte, a la misma edad $23,43 \%$ de los árboles presentaron eventos de florecimiento. Por lo tanto, se decidió realizar el análisis basado en el sitio de Tunga Norte. Sin embargo, se debe resaltar que las condiciones ambientales fueron determinantes en la precocidad de producción de flores, tomando en cuenta la edad en que fueron registrados los datos.

El resultado del criterio de ajuste del modelo linear generalizado, según la función de ligación logit se presenta en el Cuadro 2. Los valores de deviancia y Chi-cuadrado de Pearson dividido por los grados de libertad correspondientes mostraron valores cercanos a 1 . Esto, evidenció un ajuste apropiado, en ausencia de súper o sobre-dispersión, de acuerdo a Myers et al., (2002). Dado el modelo ajustado, el efecto de procedencias fue estadísticamente significativo según el análisis de desvianza (Cuadro 3). En el análisis de contrastes, la procedencia de Cowell (FDI-20411) tuvo el mayor valor promedio de florecimiento precoz, con 32,6\% de plantas con eventos florales, $39 \%$ superior al promedio total

Cuadro 2. Resultado del criterio del ajuste del modelo lineal generalizado usando la función de ligación logit.

Table 2. Result of the criteria for assessing goodness of fit using the logit link function.

\begin{tabular}{lccc}
\hline & \multicolumn{2}{c}{ Función de ligación Logit } \\
Criterio & $\mathrm{GL}^{2}$ & Valor & Valor/GL \\
\hline Desvianza & 116 & 113,823 & 0,98 \\
Desvianza escalar & 116 & 113,823 & 0,98 \\
Pearson $\chi^{2}{ }_{1}$ & 116 & 105,127 & 0,91 \\
Pearson $\chi^{2}$ escalar & 116 & 105,127 & 0,91 \\
\hline
\end{tabular}

${ }^{1}: \chi^{2}$ Chi-cuadrado.

${ }^{2}$ GL: grados de libertad.

${ }^{\prime}: \chi^{2}$ Chi-square.

${ }^{2} G L$ : degrees of freedom. 
Cuadro 3. Análisis de desvianza realizado para establecer diferencias significativas entre las procedencias de Eucalyptus cladocalyx, basado en el florecimiento.

Table 3. Analysis of deviance performed to evaluate significance differences between provenances of Eucalyptus cladocalyx, based on flowering.

\begin{tabular}{lcccc}
\hline Fuente de variación & Grados de libertad & Desvianza & Chi-cuadrado & $\mathrm{p}$ \\
\hline Intercepto & & 230,7 & & \\
Bloque & 29 & 182,0 & 48,67 & 0,0125 \\
Procedencia & 4 & 114,0 & 68,20 & $<0,0001$ \\
\hline
\end{tabular}

del ensayo. FDI-20411 no fue estadísticamente diferente de los árboles de origen chileno (FDIIllapel), que presentaron un valor promedio de 29,63\% (Cuadro 4). No obstante, esta última población no fue estadísticamente diferente de FDI-20388 y FDI-20389, debido a su alta variabilidad en el florecimiento precoz. Mora (2006) encontró que ciertas características de crecimiento de los árboles presentaron bajos valores de predicción del efecto genético de poblaciones provenientes de Cowell e Illapel. McDonald (2003) menciona que los árboles pertenecientes a la región de Eyre Peninsula, Australia, que incluye la localidad de Cowell, alcanzan un crecimiento en altura promedio menor que los árboles que crecen en otras regiones naturales. Aunque no estudiado aquí, estos antecedentes sugieren una respuesta inversa entre los caracteres crecimiento y florecimiento precoz, para esta población.

Diversos estudios han mencionado el hecho de que en ensayos de procedencias de una determinada especie que presenta una gran distribución geográfica, generalmente se evidencia una significativa variación genética en diversas características fisiológicas y morfológicas (Beniwal et al., 1995; Mulatya et al., 2002). Eucalyptus cladocalyx es un árbol con una restringida distribución en la localidad de Southern Flinders Ranges, sur de Australia (Ruthrof et al., 2003). A pesar de esta restringida distribución, en el presente estudio, la variabilidad entre procedencias mostró ser altamente significativa. Este resultado puede ser importante para la continuidad del programa de mejoramiento con E. cladocalyx bajo las condiciones áridas del norte de Chile. Estudios previos han demostrado que el éxito de los programas de mejoramiento de árboles en áreas con déficit hídrico, depende de la selección previa de procedencias ya adaptadas a las condiciones locales de suelo y clima (Broadmeadow et al., 2005).

Dado los resultados descritos anteriormente,

Cuadro 4. Resultado de la prueba de contrastes realizado para analizar las diferencias estadísticas entre los valores promedios de florecimiento.

Table 4. Results of the contrasts test performed to analyze statistical differences between mean values of flowering.

\begin{tabular}{lccccc}
\hline Contrastes & Grados de libertad & $\begin{array}{c}\text { Chi cuadrado } \\
\chi^{2}\end{array}$ & $\mathrm{p}$ & Procedencia & Promedio \\
\hline FDI-20388 - FDI-20411 & 1 & 5,19 & 0,0228 & FDI-20388 & $25,00 \mathrm{~b} 1$ \\
FDI-20388 - FDI-20389 & 1 & 0,01 & 0,9164 & FDI-20411 & $32,60 \mathrm{a}$ \\
FDI-20388 - FDI-20267 & 1 & 46,90 & $<0,0001$ & FDI-20389 & $24,62 \mathrm{~b}$ \\
FDI-20388 - FDI-Illapel & 1 & 0,38 & 0,5366 & FDI-20267 & $5,24 \mathrm{c}$ \\
FDI-20411 - FDI-20389 & 1 & 4,40 & 0,0358 & FDI-Illapel & $29,63 \mathrm{ab}$ \\
FDI-20411 - FDI-20267 & 1 & 65,69 & $<0,0001$ & & \\
FDI-20411 - FDI-Illapel & 1 & 0,34 & 0,5584 & & \\
FDI-20389 - FDI-20267 & 1 & 38,71 & $<0,0001$ & & \\
FDI-20389 - FDI-Illapel & 1 & 0,42 & 0,5162 & & \\
FDI-20267 - FDI-Illapel & 1 & 23,15 & $<0,0001$ & &
\end{tabular}

${ }^{1}$ Promedios seguidos por letras distintas son significativamente diferentes entre sí.

${ }^{I}$ Means followed by different letter are significantly different. 
se consideró la procedencia de Cowell como la población seleccionada para el estudio de la base genética intra-poblacional. Consecuentemente, el análisis genético de árboles individuales se conformó por diez familias provenientes de polinización abierta, considerando el sitio de Tunga Norte.

Las distribuciones marginales a posteriori de los componentes de varianza y heredabilidad se presentan en la Figura 1. Las estimaciones de los componentes de varianza, basados en el promedio, y heredabilidad, basado en el promedio, mediana y moda, son mostradas en el Cuadro 5. Las cadenas generadas por el algoritmo de Gibbs evidenciaron un diagnóstico positivo a la convergencia, de acuerdo con la prueba de Heidelberger y Welch (1983), para los componentes de varianza y heredabilidad, considerando 550 mil muestras, incluyendo 50 mil de descarte (burn-in). En inferencia Bayesiana usando el algoritmo Gibbs, el período de descarte es necesario para reducir los efectos de los valores iniciales (Wright et al., 2000) y obtener inferencias confiables de los parámetros de interés.

La característica de florecimiento precoz mostró ser altamente heredable (Cuadro 5), con un valor medio de heredabilidad de 52,29\%. La media, mediana y moda de la heredabilidad mostraron ser semejantes, evidenciando una relativa simetría en las estimaciones de distribución a posteriori (Van Tassell et al., 1998) (Figura 1).

En la selección de árboles individuales dentro de la mejor procedencia, dos criterios se consideraron en función de la intensidad de selección. Un primer criterio fue basado en una intensidad de selección para conformar una población de producción de semillas, a un nivel operativo o de corto plazo, y el segundo criterio fue basado en la selección de árboles para conformar una población de mejoramiento, o estrategia de mediano plazo. Basado en la estrategia de selección de corto plazo, es posible obtener una importante ganancia genética
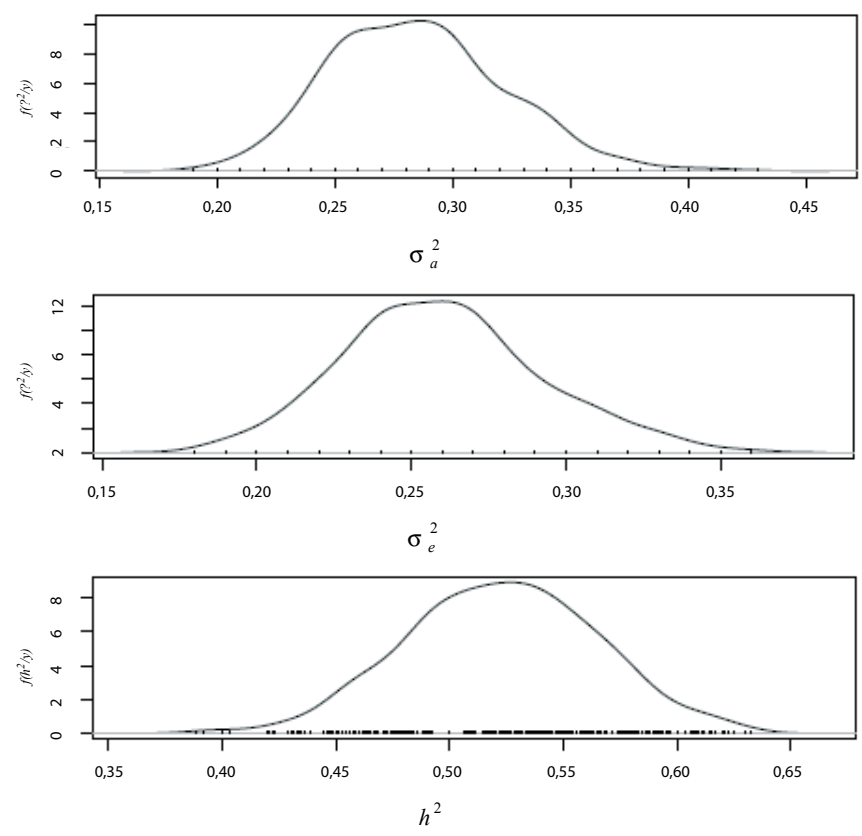

Figura 1. Distribución a posteriori de la varianza aditiva $\left(\sigma_{a}^{2}\right)$, varianza residual $\left(\sigma_{e}^{2}\right)$ y heredabilidad $\left(h^{2}\right)$, para el florecimiento precoz de árboles provenientes de la localidad de Cowell, Australia.

Figure 1. Posterior distributions for additive $\left(\sigma_{a}^{2}\right)$ and residual variances $\left(\sigma_{e}^{2}\right)$, and heritability estimates $\left(h^{2}\right)$ for flowering precocity of the trees from Cowell, Australia. 
Cuadro 5. Componentes de varianza, heredabilidad, número de árboles seleccionados (NAS), número de familias seleccionadas (NFS), y ganancia genética predicha (GG) en función de la intensidad de selección (IS), basado en el florecimiento precoz dentro de la procedencia de Cowell, Australia.

Table 5. Variance components, heritability, number of selected trees (NAS), number of selected families (NFS), and predicted genetic gain $(G G \%)$ in function of intensity of selection (IS\%), according to flowering precocity within Cowell provenance, Australia.

\begin{tabular}{|c|c|c|c|c|c|c|c|}
\hline \multicolumn{2}{|l|}{ Varianza } & \multicolumn{2}{|c|}{ Heredabilidad } & \multirow{2}{*}{$\begin{array}{l}\text { IS } \\
\% \\
8\end{array}$} & \multirow{2}{*}{$\begin{array}{c}\text { NAS } \\
24\end{array}$} & \multirow{2}{*}{$\begin{array}{c}\text { NFS } \\
5\end{array}$} & \multirow{2}{*}{$\begin{array}{c}\text { GG } \\
\%\end{array}$} \\
\hline Aditiva & 0,2851 & Media & 0,5229 & & & & \\
\hline Residual & 0,2598 & Mediana & 0,5222 & 15 & 45 & 9 & 36,06 \\
\hline Fenotípica & 0,5450 & Moda & 0,5260 & & & & \\
\hline
\end{tabular}

predicha de 39,72\% (Cuadro 5). A su vez, en la población de mejoramiento se alcanzaría una ganancia aproximada de 36,06\%. El número de familias consideradas en la selección varió de 5 a 9, dependiendo de la estrategia adoptada.

Laseleccióngenotípicabasadaenelflorecimiento precoz tiene por finalidad recuperar lo más rápido posible el valor de la inversión efectuada por el agricultor. Cabe destacar que los árboles con presencia de eventos florales mostraron en su mayoría una relativa abundancia de yemas, o de cualquier formación posterior a ella. Aunque empíricamente, este factor también merece ser destacado. Porter (1978) determinó que la producción de miel de Eucalyptus en zonas áridas depende del número de colmenas en un sitio determinado, del tiempo de duración de la cosecha, la precipitación, la temperatura durante y antes de la cosecha, y de la abundancia de floración. Wiltshire et al., (1998) encontraron un moderado control genético aditivo en el florecimiento precoz en familias provenientes de polinización abierta de E. risdonii y $E$. tenuiramis, con valores de heredabilidad variando de 0,31 a 0,41. Chambers et al., (1997) encontraron una moderada a alta heredabilidad en árboles de 4 años de edad de E. globulus, en Tasmania, estimando valores de 0,47 a 0,59. En el presente estudio, el valor relativamente alto obtenido para la heredabilidad individual, o grado de control genético aditivo, indica que métodos simples de selección pueden ser implementados. Tal información es muy útil para el inicio del programa de mejoramiento de árboles de E. cladocalyx usando esta población (Cowell) en la región de Coquimbo.

Los eventos asociados al primer florecimiento de las poblaciones de E. cladocalyx, consideradas en este estudio, mostraron ser dependientes del efecto de procedencias y del ambiente, con una incipiente floración precoz en el sector litoral de la Provincia de Choapa.

Las variaciones entre procedencias y el control genético aditivo evidenciado en la población de Cowell, en el sector de Tunga Norte, permitieron obtener importantes ganancias genéticas, cuya finalidadesquelos agricultores puedan recuperar al corto plazo los costos de establecimiento de la plantación con E. cladocalyx, siempre que los árboles seleccionados sean utilizados para complementar la actual producción de miel, la que es basada esencialmente en la valiosa flora nativa regional.

\section{Resumen}

Se analizó la variabilidad en el florecimiento de cinco poblaciones de Eucalyptus cladocalyx establecidos en la Región de Coquimbo. Se consideró al florecimiento como una respuesta binaria medida a los 30 meses de edad. Por lo tanto, se ajustó un modelo linear generalizado para analizar el efecto de procedencias. Posteriormente, se estudió la variabilidad genética dentro de la mejor procedencia, a través de inferencia Bayesiana, vía algoritmo de Gibbs. Inicialmente, se consideraron dos sitios, Hacienda Caracas y Tunga Norte, sector costero e interior de la Provincia de Choapa, respectivamente. En el sector costero, sólo un $1,33 \%$ de los árboles presentaron eventos de florecimiento, mientras que en Tunga Norte, este valor fue de $23,43 \%$. Debido a este resultado, se consideró solo el sitio interior. El efecto de procedencias fue 
estadísticamente significativo $(\mathrm{p}<0,01)$ siendo Cowell (codificada FDI-20411) la población que presentó una mejor respuesta relativa a los eventos de floración, aunque no fue diferente de los árboles de origen nacional (FDI-Illapel). Sin embargo, esta última población presentó una alta variabilidad, teniendo una respuesta media similar estadísticamente a otras procedencias. El florecimiento analizado dentro de la población de Cowell, población seleccionada, mostró ser altamente heredable $\left(\mathrm{h}^{2}=52,29 \%\right)$. La ganancia genética predicha, considerando distintas estrategias de selección, varió entre 36 y $40 \%$. En función de estos resultados, es posible que el agricultor pueda recuperar al corto plazo el costo de establecimiento de la plantación de E. cladocalyx, siempre que los árboles seleccionados sean utilizados con fines melíferos.

Palabras clave: Ambientes áridos, árboles multipropósito, eventos de florecimiento, mejoramiento, procedencias.

\section{Agradecimientos}

Se agradece a la Coordinación de Perfeccionamiento del Personal de Nivel Superior (CAPES), Ministerio de Educación de Brasil, por la beca doctoral concedida al primer autor, y al Fondo de Desarrollo e Innovación (FDI), del Ministerio de Economía de Chile, por el aporte financiero. Especiales agradecimientos por la colaboración recibida por parte de los agricultores de la Comunidad Agrícola de Tunga Norte y la Hacienda Agrícola Caracas, en la Región de Coquimbo, Chile.

\section{Literatura citada}

Avila, G., M. Gómez, A.M. Mujica y G. Montenegro. 1993. La flora nativa sustentadora de colmenas de Apis mellifera en Pichidangui IV Región de Chile. Ciencia e Investigación Agraria 20:119125.

Beniwal, R.S., O.P. Toky, and P.K. Sharma. 1995. Genetic variability in symbiotic nitrogen fixation between provenances of Acacia nilotica (L.) Willd. ex Del. Genetic Resources and Crop Evolution 42:7-13.

Broadmeadow, M.S.J., D. Ray, and C.J.A. Samuel. 2005. Climate change and the future for broadleaved tree species in Britain. Forestry 78:145-161.

Bonilla, C., C. Bonomelli y G. Urrutia. 2000. Funciones de crecimiento basadas en el contenido de humedad en el suelo para plantaciones jóvenes de eucalipto. Ciencia e Investigación Agraria 27:132-190.

Bonomelli, C. y D. Suárez. 1999. Fertilización del eucalipto. 1. Efecto sobre la acumulación de biomasa. Ciencia e Investigación Agraria 26:1-10.

Chambers, P.G.S., B.M. Potts, and P.A. Tilyard. 1997. The genetic control of flowering precocity in Eucalyptus globulus ssp. globulus. Silvae Genetica 46:207-214.

Cowles, M.K., N. Best, and K. Vines. 1995. Convergence diagnostics and output analysis. MRC Bioestatistics Unit, London, UK, 127 pp.

Crane, E., P. Walter, and R. Day. 1984. Directory of Important World Honey Sources. International Bee Research Association, London, UK, 384 pp.

Dos Santos, A. L. y F. Mora. 2007. Análisis experimental de tratamientos floculantes de residuos orgánicos derivados de la producción porcina. Ciencia e Investigación Agraria 34:4754.

Fuentes, E., G. Montenegro, P. Rundel, M. K. Arroyo, R. Ginocchio, and F. Jaksic. 1995. The functions of biodiversity in the Mediterraneantype ecosystem of Central Chile. Pages 185-232, In: G. Davies and D. Richardson (eds.). Mediterranean-types Ecosystems. The Functions of Biodiversity. Springer-Verlag. New York, USA.

Geldres, E. y J.E. Schlatter. 2004. Crecimiento de las plantaciones de Eucalyptus globulus sobre suelos rojo arcillosos de la provincia de Osorno, Décima Región. Bosque 25:95-101.

Geldres, E., V. Gerding y J. E. Schlatter. 2006. Biomasa de Eucalyptus nitens de 4-7 años de edad en un rodal de la X Región, Chile. Bosque 27:223-230.

Gleadow, R.M., W.J. Foley, and I.E. Woodrow. 1998. Enhanced $\mathrm{CO}_{2}$ alters the relationship between photosynthesis and defence in cyanogenic Eucalyptus cladocalyx F. Muell. Plant, Cell and Environment 21:12-22.

Hanks, L.M., T.D. Paine, J.G. Millar, and J.L. Hom. 1994. Variation among Eucalyptus species in resistance to eucalyptus longhorned borer in Southern California. Entomologia Experimentalis et Applicata 74:185-194.

Heidelberger, P., and P.D. Welch. 1983. Simulation run length control in the presence of an initial transient. Operations Research 31:1109-1114.

Jorquera, P. 1998. Taladrador bajo la lupa. Revista Chile Forestal. 23:26-27.

Manikis, I., and A. Thrasivoulou. 2001. The relation 
of physicochemical characteristics of honey and the crystallization sensitive parameters. Apiacta 36:106-112.

Mcdonald, M.W., M. Rawlings, P.A. Butcher, and J.C. Bell. 2003. Regional divergence and inbreeding in Eucalyptus cladocalyx (Myrtaceae). Australian Journal of Botany 51:393-403.

Montenegro, G., R. Pizarro, G. Avila, R. Castro, C. Ríos, O. Muñoz, F. Bas y M. Gómez. 2003. Origen botánico y propiedades químicas de las mieles de la región mediterránea árida de Chile. Ciencia e Investigación Agraria 30:161-174.

Mora, F. 2004. Análisis preliminar de la heredabilidad e interacción genotipo-ambiente del crecimiento, en dos ensayos de progenie de Eucalyptus cladocalyx (F. Muell) establecidos en la zona árida de Chile. Tesis de Magíster, Universidad de La Serena, Chile. 71 pp.

Mora, F. 2006. Heredabilidad y valor genético (REML/BLUP) en genotipos de un eucalipto tolerante a la sequía, en el norte de Chile. Ciencia Florestal 16:145-151.

Mora, F. y E. Arnhold. 2006. Inferencia Bayesiana y metodología de modelos lineales mixtos aplicados al mejoramiento del maíz. Ciencia e Investigación Agraria 33:217-223.

Mora, F. y S. Perret. 2002. Eucalyptus cladocalyx en la Región de Coquimbo: Especie maderera para el negocio frutícola. Revista Tierra Adentro 46:28-29.

Mora F., O. Pupim-Júnior y C.A. Scapim. 2007. Predicción del efecto de cultivares de algodón en la presencia de interacción genotipo-ambiente. Ciencia e Investigación Agraria 34:11-19.

Mulatya J.M., J. Wilson, C.K. Ong, J.D. Deans and J.I. Sprent. 2002. Root architecture of provenances, seedlings and cuttings of Melia volkensii: implications for crop yield in dryland agroforestry. Agroforestry Systems 56:65-72.

Myers, R.H., D.C. Montgomery, and G.G. Vining. 2002. Generalized Linear Models, with Applications in Engineering and the Sciences. John Wiley and Sons Press, New York, USA, $342 \mathrm{pp}$.

Nelder, J.A., and R.W.M. Wendderburn. 1972. Generalized linear model. Journal of the Royal
Statistical Society A 135:370-384.

Porter, J.W. 1978. Relationships between flowering and honey production of red ironbark, Eucalyptus sideroxylon (A. Cunn.) Benth., and climate in the Bendigo district of Victoria. Australian Journal of Agricultural Research 29:815-829.

Prado, J. A. y S. Barros. 1989. Eucalyptus: Principios de Silvicultura y Manejo. INFOR-CORFO, Santiago, Chile. 199 pp.

Ruthrof, K.X., W.A. Loneragan, and C.J. Yates. 2003. Comparative population dynamics of Eucalyptus cladocalyx in its native habitat and as an invasive species in an urban bushland in south-western Australia. Diversity and Distributions 9:469-483.

Squeo, F.A., Y. Tracol, D. López, J.R. Gutiérrez, A.M. Cordova, and J.R. Ehleringer. 2006. ENSO effects on primary productivity in Southern Atacama desert. Advances in Geosciences 6:273-277.

Van Tassell, C.P., and L.D. Van Vleck. 1996. Multiple-trait Gibbs sampler for animal models: flexible programs for Bayesian and likelihoodbased (co)variance component inference. Journal of Animal Science 74:2586-2597.

Van Tassell, C.P., L.D. Van Vleck, and K.E. Gregory. 1998. Bayesian analysis of twinning and ovulation rates using a multiple-trait threshold model and Gibbs sampling. Journal of Animal Science 76:2048-2061.

Wiltshire, R.J.E., J.B. Reid, and B.M. Potts. 1998. Genetic control of reproductive and vegetative phase change in the Eucalyptus risdonii-E. tenuiramis complex. Australian Journal of Botany 46:45-63.

Woodrow, I.E., D. Slocum, and R.M. 2002. Gleadow. Influence of water stress on cyanogenic capacity in Eucalyptus cladocalyx. Australian Journal of Plant Physiology 29:103-110.

Wright, D.R., H.S. Stern, and P.J. Berger. 2000. Comparing traditional and Bayesian analyses of selection experiments in animal breeding. Journal of Agricultural, Biological, and Environmental Statistics 5:240-256. 